\title{
Optimal design of a class of symmetric plane frameworks of least weight
}

\author{
Reply to the paper: Vázquez Espí and Cervera Bravo (2011) On the solution of the three forces \\ problem and its application in optimal designing of a class of symmetric plane frameworks of least \\ weight. Discussion on the Michell class concept
}

\author{
T. Sokół • T. Lewiński
}

Received: 27 July 2011 / Revised: 1 August 2011 / Accepted: 4 August 2011 / Published online: 27 September 2011

(C) The Author(s) 2011. This article is published with open access at Springerlink.com

\begin{abstract}
The aim of the paper is to place the recent results on the Michell-like problem of transmitting two vertical forces to two fixed hinge supports in the context of C. Maxwell's result and to provide answers to the remarks by Vázquez Espí and Cervera Bravo (Struct Multidisc Optim, 2011) published in their Discussion Paper: On the solution of the three forces problem and its application in optimal designing of a class of symmetric plane frameworks of least weight. Discussion on the Michell class concept.
\end{abstract}

Keywords Topology optimization of trusses .

Michell trusses - Minimum weight design .

Plastic layout optimization $\cdot$ Linear programming

\section{Introduction}

The paper by Vázquez Espí and Cervera Bravo (2011) (called further: Discussion) refers mainly to Section 5 of Sokół and Lewiński (2010) including both numerical and

Corrigendum to the paper: Sokół T, Lewiński T (2010) On the solution of the three forces problem and its application in optimal designing of a class of symmetric plane frameworks of least weight. Struct Multidisc Optim 42:835-853

In Table 1 the columns labeled as $x_{\mathrm{D}} / d, y_{\mathrm{D}} / d$ should be labeled by $x_{\mathrm{D}} / L, y_{\mathrm{D}} / L$, respectively. The present authors express their gratitude to M. Vázquez Espí and J. Cervera Bravo to note this mistake.

T. Sokół $(\bowtie) \cdot$ T. Lewiński

Department of Structural Mechanics and Computer

Aided Engineering, Faculty of Civil Engineering,

Warsaw University of Technology,

Al. Armii Ludowej 16, 00-637 Warsaw, Poland

e-mail: t.sokol@il.pw.edu.pl analytical solution to the Michell-like problem of transmitting two vertical forces to two fixed hinges. Both forces are located symmetrically with respect to the midperpendicular of the section linking the hinges. The members of the unknown structures should be stressed up to given limits $\pm \sigma_{p}$ and should lie above the line linking the supports. Since this problem has been previously discussed in the remarkable paper by McConnel (1974) including the numerical solutions comparing favorably with those obtained by the present authors (2010) and by Sokół (2011) with using much better software and hardware, one can name this problem shortly: McConnel's problem.

McConnel's problem is a natural extension of the Michell solution concerning one force applied in the middle between two fixed hinges. The formulations of the selected two McConnel's problems and the latter problem, as well as the sketches of their solutions found in Sokół and Lewiński (2010) are shown in Fig. 1.

Let us consider now a seemingly similar problem for which one (or both) supports are sliding, see Fig. 2.

We note that problem (c) in Fig. 2 has already been solved by Michell (1904), while the exact solutions to problems $(a, b)$ have been found only recently by Sokół and Lewiński (2011). These solutions form a certain subclass of the four forces problem in which they form a symmetric system of four parallel forces of equal magnitude.

If we extend the feasible domain to the whole plane the exact layouts become independent of one or both supports being sliding or not. This follows from one of the symmetry propositions of Rozvany (2011). The exact layouts have now empty regions in the shapes of squares, see Fig. 3, cf. Sokół and Rozvany $(2011,2012)$.

Having at our disposal all these exact solutions we can easier assume our attitude to the remarks of Vázquez Espí and Cervera Bravo (2011). 


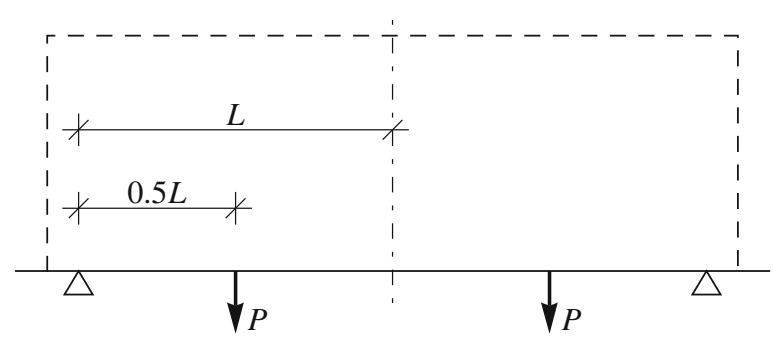

(a)
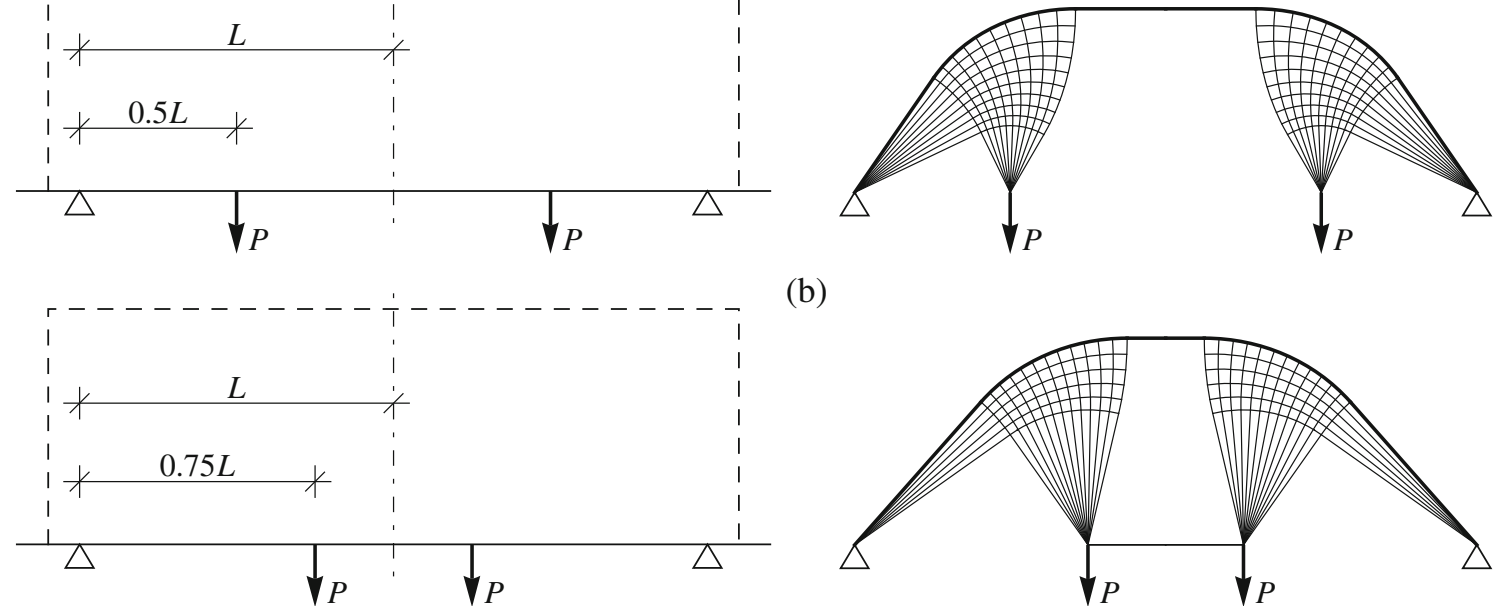

(b)
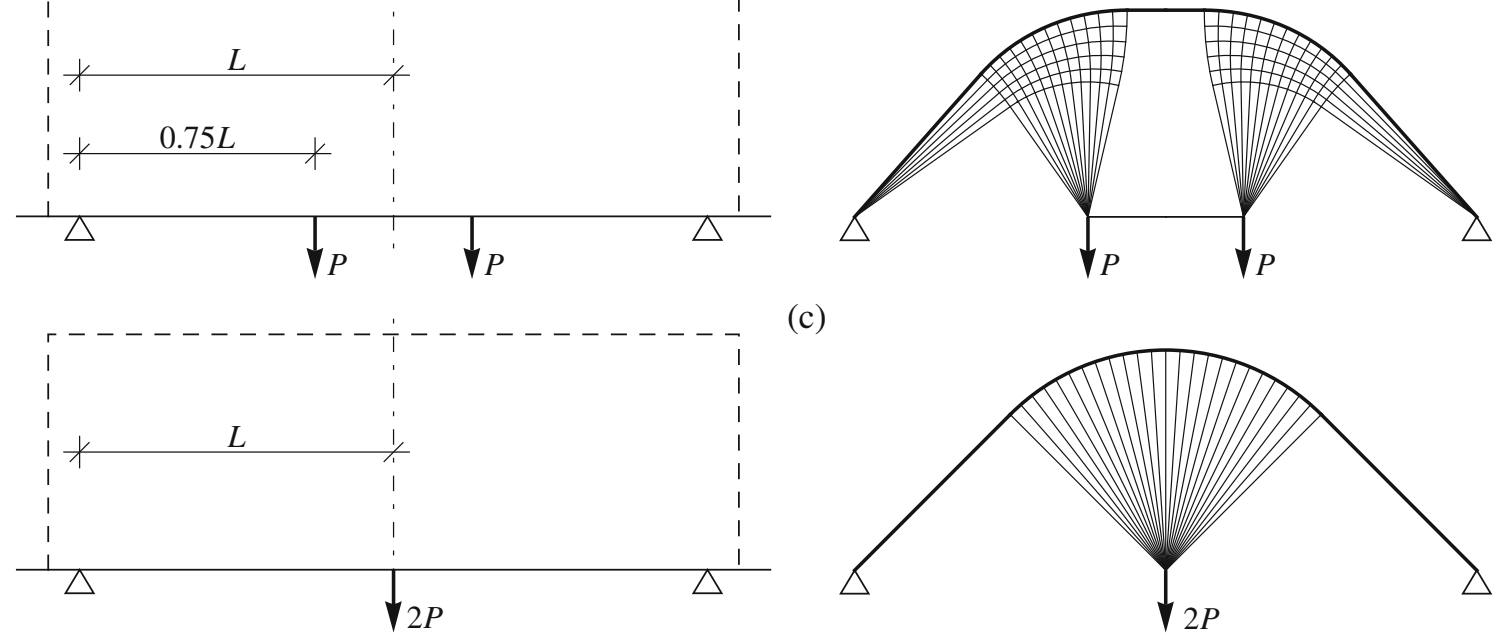

(c)

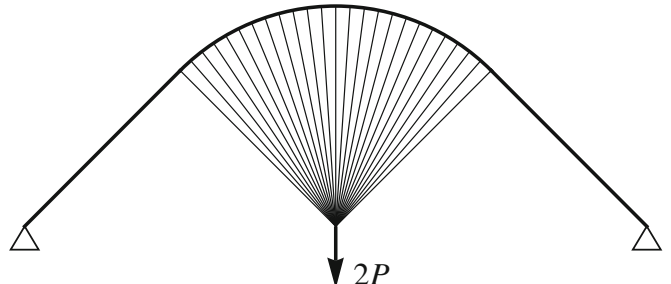

Fig. 1 Formulations and exact solutions of the McConnel problem (a, b) and Michell’s problem (c), (after Sokół and Lewiński 2010)

\section{On Maxwell's theorem and its relation to Michell's results}

Vázquez Espí and Cervera Bravo (2011) draw our attention to the role of Clerk Maxwell's (1870, see pages: 175-177) results which was the inspiration of Michell's (1904) work. This Maxwell's result is thoroughly described in Ch.8 of the book by Cox (1965, pp.80-84), is referred to in Hemp's book (1973, p.72, Eq (4.10)) and discussed by McConnel (1974, p.886, Eq (5)). We shall not repeat this result here. It seems that the Authors of the Discussion pay too much attention to this result, since its direct application in the minimum weight design of trusses confines to a rather narrow class of trusses for which the virtual strain is identical in all bars. Let us repeat after Cox (1965, p.86): "the need for this limitation arises from the insufficiency of the Clerk Maxwell lemma as a guide to the best layout". Let us stress: it is Michell who noted this insufficiency and introduced a new class of layouts much more useful for finding a wide class of structures of least weight. Let us note: the Michell class encompasses all Maxwell-like optimal solutions (i.e. the optimal solutions which can be inferred from the result (4.10) in Hemp (1973)). Hence there is no need now to consider Maxwell's solutions separately.

\section{On problem (3) in the Discussion}

By Maxwell's theorem the quantity $\mathcal{M}$ in Eq (3) of the Discussion depends only on the forces applied, hence its variation over all feasible layouts vanishes: $\delta \mathcal{M}=0$ (we interpret here $\partial$ as $\delta$ ). Hence the latter condition is redundant if the equilibrium conditions are involved-and this should be the case, which has not been indicated in problem (3) of the Discussion. By replacing $\delta \mathcal{M}=0$ with the equilibrium equations, we rearrange problem (3) to the simple formulation of minimizing the weight but without additional assumptions concerning the stress level. Hence this formulation is incomplete. That is why it is difficult to discuss the formulation (3) in the sequel.

\section{On remarks in Section 3 of the Discussion concerning the half-plane problem}

The Authors of the Discussion claim that the aim of optimization should not be the weight of the structure; this weight functional should be augmented by a term measuring the cost of some forces. The Authors suggest in this respect that the new functional is naturally inferred from Maxwell's 


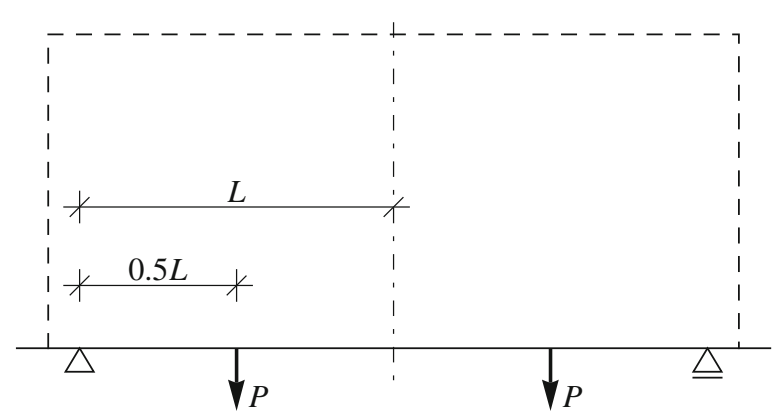

(a)

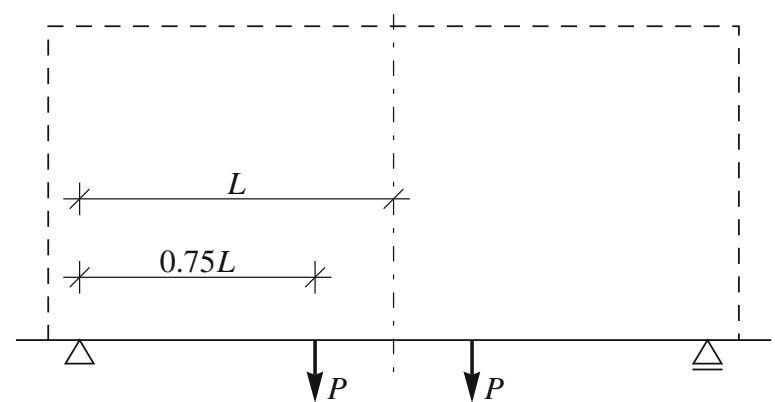

(b)
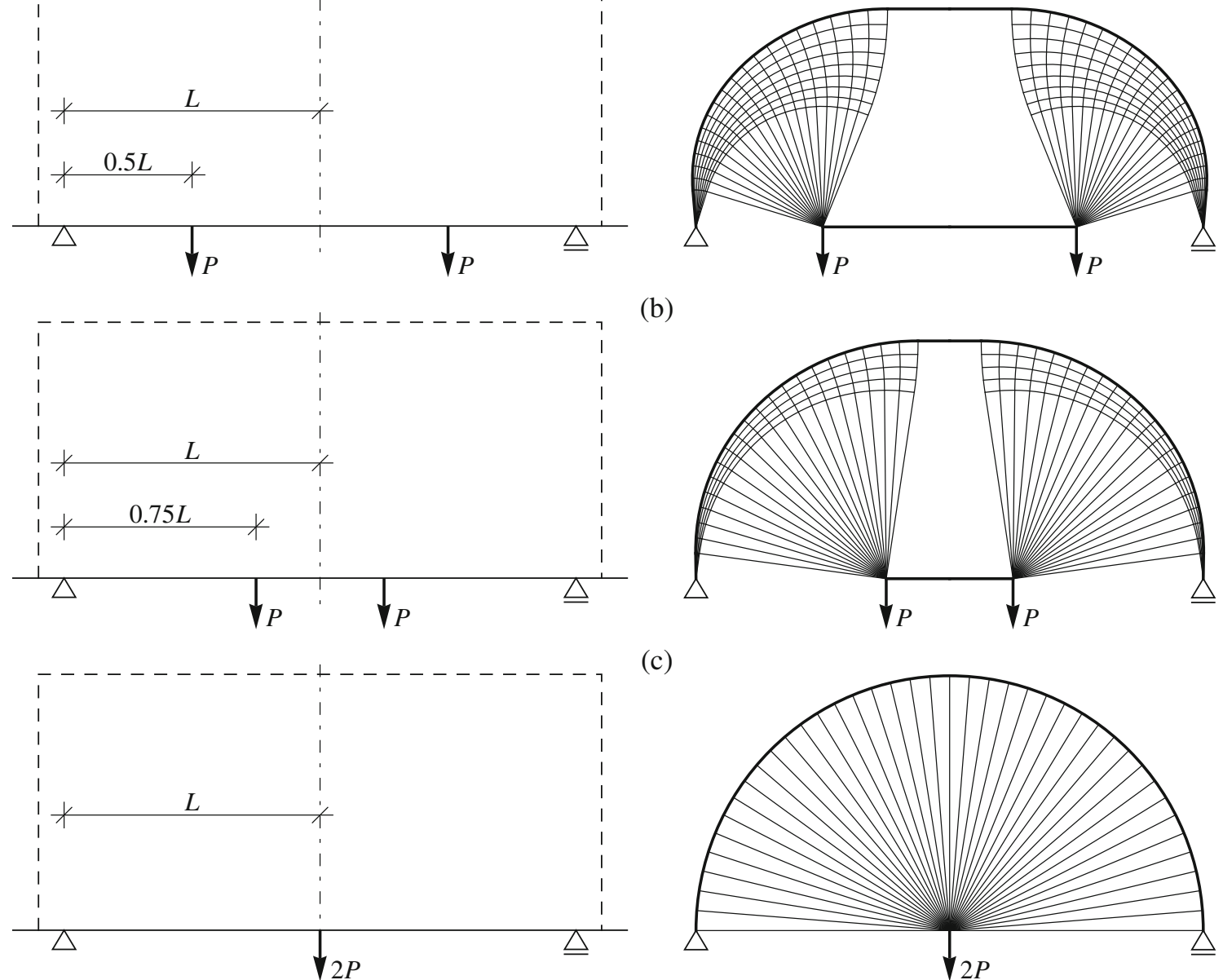

(c)

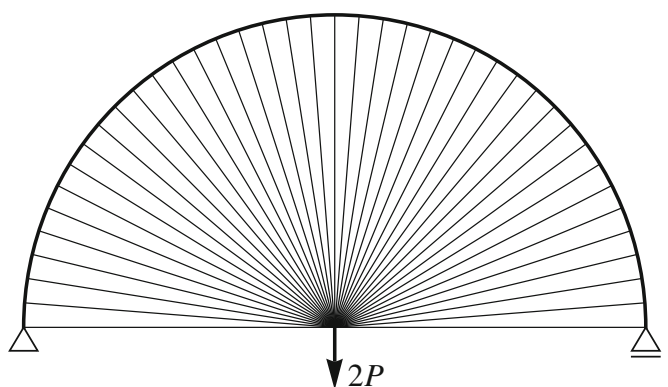

Fig. 2 Formulations and exact solutions for problems with one sliding support (after Sokół and Lewiński 2011)

equality (see Eq (4.10) in Hemp (1973)). This new functional (or merit function) should be defined as the sum of the weight plus a term proportional to the values of some reactions. The Authors focus their attention on the problem of Section 5.1 in Sokół and Lewiński (2010). The Authors of the Discussion propose to consider a new problem: minimize the merit function (it is a functional over possible layouts):

$J=V+\alpha H, \quad \alpha=2 L / \sigma_{p}$

where $V$ is the volume of the structure and $H$ is the horizontal reaction as in Fig. 18 in Sokół and Lewiński (2010). Although the original texts by Maxwell have been at the present authors' disposal, as well as the mentioned above works by Cox, Hemp and McConnel in which Maxwell's results are described in a contemporary language (let us emphasize here that the main result of Maxwell was described in words-p.175 in Maxwell (1870)_-and not in mathematical symbols) the present authors cannot confirm that the functional $J$ has its origin in Maxwell's papers.

The present authors suspect that the solution to the problem: minimize $J$ over all plane structures transmitting two given forces to two given hinges, as in Fig. 18 of Sokół and Lewiński (2010), has probably never been considered and its solutions are unknown. Note that each solution must depend on the $\alpha$ coefficient. The value of $\alpha$ assumed as in (1) does not follow from the engineering practice. The term $\alpha H$ can be interpreted as the cost of the supports and the value of $\alpha$ should be fixed upon assuming the method of constructing the support. Thus the problem of minimizing $J$ has one-parameter family of solutions - indexed by $\alpha$.

Below Table 1 in the Discussion the Authors suggest that the upper structure in Fig. 1 of the Discussion-with an additional horizontal bar linking the supporting pointsrepresents the solution to the problem of minimizing $J$ in case of the feasible domain being the half-plane. The 
present authors would like to stress here that this figure bears no relation to the true solution of this problem and publishing it is very misleading. Consequently, the results called S\&L of Table 1 under the title "Maxwell problem" should not have been published as both misleading and incorrect. The lower layout of Fig. 1 of the Discussion has been found by the Authors and it is probably an approximate solution to the problem of minimizing $J$. If this is the case, this numerical solution cannot be compared with the solutions published in Sokół and Lewiński (2010) and the layouts published there cannot be transformed to the form of the solution to any problems of minimizing $J$. It is possible that for some values of $\alpha$ the problem of minimizing $J$ reduces to the problem of weight minimization for the case
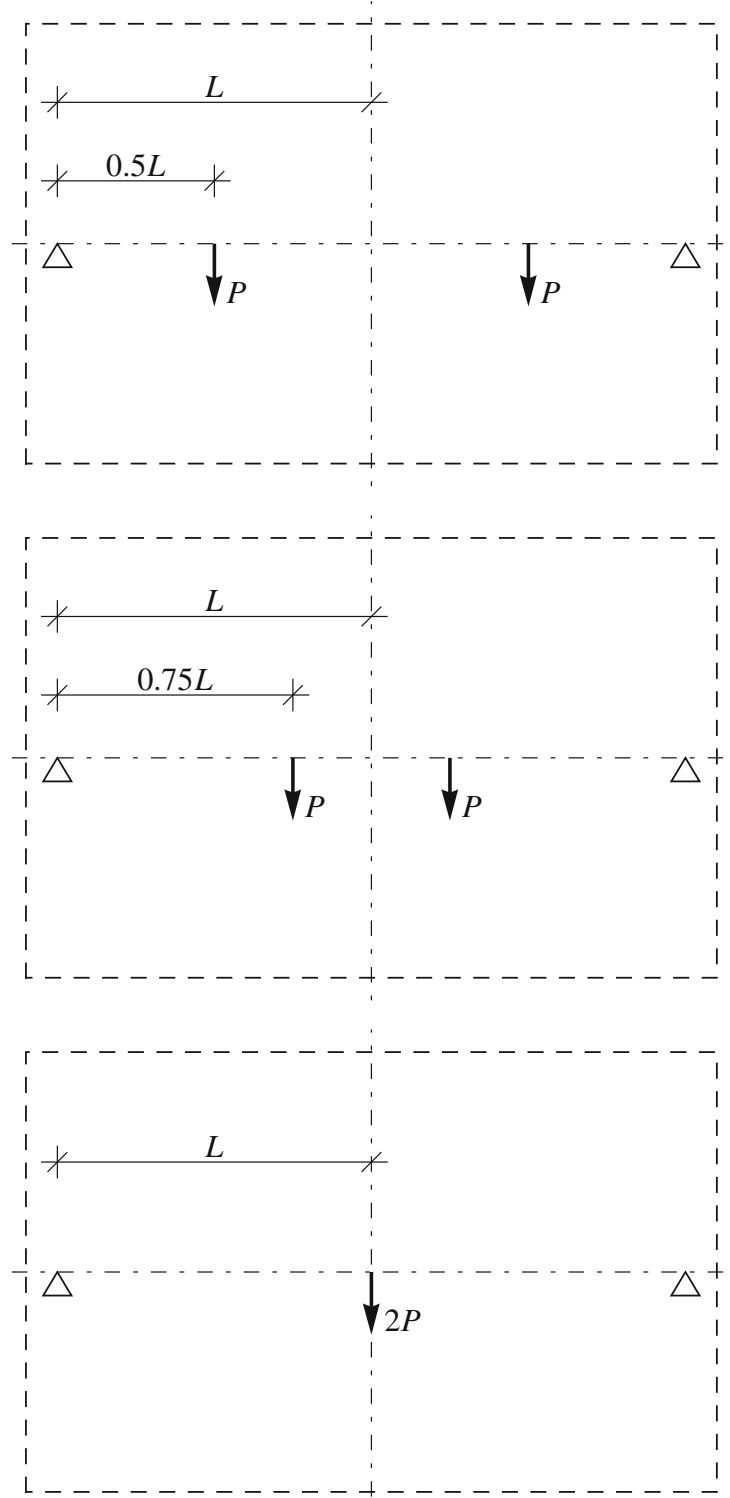

of both supports being sliding in the horizontal direction and for the case of the feasible domain being the half-plane, see Fig. 2 of the present paper. In this problem $H$ vanishes and $J$ equals the weight. Yet the layout in Fig. 1 (lower figure in the Discussion) is different from the solutions shown in Fig. 2 of the present paper.

\section{On remarks formulated in the Discussion concerning the full plane problem}

Further remarks of Section 3 of the Discussion refer to the problem of Fig. 3 of the present paper. This problem has not been considered in Sokół and Lewiński (2010) as the

(a)

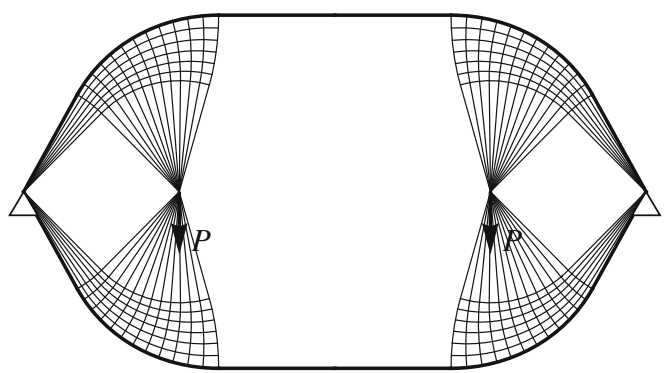

(b)

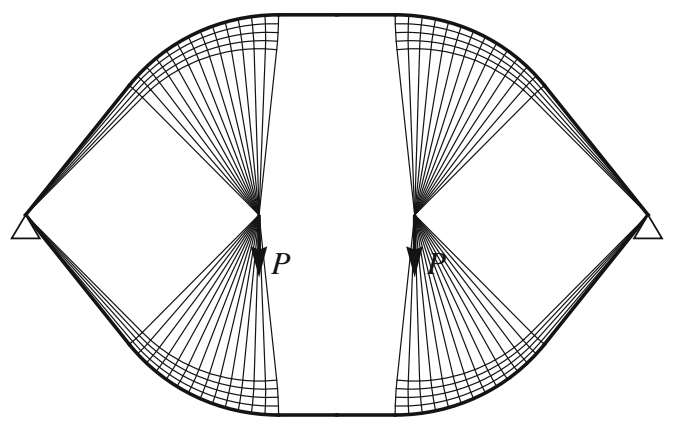

(c)

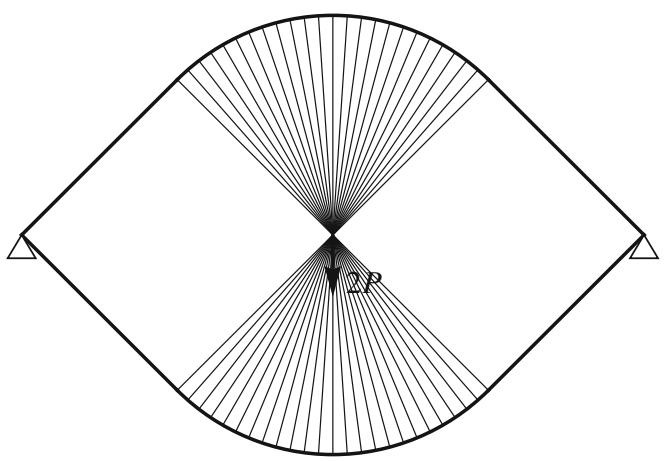

Fig. 3 Formulation and exact layouts for the case of the feasible domain being the whole plane (after Sokół and Rozvany 2011). The supports may be sliding in the horizontal direction with no effect on the form of the solutions in all cases shown 
Authors of the Discussion suggest. It has been recently solved in Sokół and Rozvany $(2011,2012)$. Let us emphasize that the solutions shown in Fig. 3 remain the same if one of supports is allowed to slide horizontally.

The characteristic feature of the solutions of Fig. 3 is that the empty regions between the vertical reactions and the vertical forces applied assume the form of squares. The solutions of Fig. 3 have been constructed by using analytical method of Sokół and Lewiński (2010) and justified by the numerical method of Sokół (2011). The upper figure in Fig. 2 of the Discussion should not be labeled by our names. This figure has nothing to do with our work of 2010.

Since here $H=0, J$ stands for the weight and the problem stated by the Authors is equivalent to Michell's problem. Thus the numerical solution in Fig. 2 (lower figure) of the Discussion is very approximate and, as highly deviated from the exact one, is misleading. The net presented in Fig. $2 b$ in Discussion does not satisfy the orthogonality requirements. For example, the empty rhomboidal regions should be square shaped.

\section{On numerical results of Table 1 in the Discussion}

As was discussed earlier the Authors of the Discussion incorrectly interpreted and enhanced the results from Sokół and Lewiński (2010). In their Table 1 the abbreviation "S\&L" suggests that these results are taken from our paper and moreover that the result obtained by using simulated annealing search are better than analytical ones. The comparison of the results of the problems in Figs. 1, 2, 3a (for $\xi=d / L=0.5$ ) are summarized in Table 1 below. This comparison is made under the assumption that the Authors of the Discussion did not consider the problem of minimizing $J$, but simply the weight minimization.

The results of Table 1 of the Discussion, repeated in column 5 of Table 1 above, show that the problem of Fig. 1a of the present paper had been misinterpreted. Probably, Vázquez Espí and Cervera Bravo studied only the problems of Figs. $2 \mathrm{a}$ and $3 \mathrm{a}$, as we guess by comparing their results with exact values of the volume. The results obtained by using the computer program developed in Sokół (2011) turn out to be more accurate then those found by the simulated annealing method. Let us emphasize that the sole comparison of the volumes is not sufficient. The numerical layouts found in Sokół and Lewiński $(2010,2011)$ and Sokół and Rozvany $(2011,2012)$ compare favorably with analytical results reported in the Introduction of the present paper. In contrast, the numerical predictions of bar layouts shown in Figs. 1 and 2 of the Discussion differ essentially from the analytical results.

\section{On construction of the virtual displacement field}

In Section 4 of the Discussion the Authors indicate that the analytical solutions in Fig. 1 here and in Fig. 20 of Sokół and Lewiński (2010) had not been substantiated by construction of the virtual displacement (and strain) fields satisfying the known Michell criteria of optimality. This is true: up till now we have not constructed these fields in the empty regions of the feasible domain. Some hints of how to construct the virtual strain fields satisfying all Michell's conditions, including kinematic admissibility of the relevant virtual displacement field can be found in Chan (1975). We do hope to solve this mathematical problem soon. We know only that in some parts in the central empty region a rigid body motion takes place, where all virtual strain components vanish.

Let us note that all solutions shown in the present paper differ considerably from the better known cantilever-like solutions. In the latter problems the condition of vanishing virtual displacements along the feasible support yields Hencky nets of geometry independent of the loading applied (if assuring unequal signs of stress resultants in two families of bars). In these solutions the Hencky nets are independent of the loading, so long as it is admissible. In the solutions of Fig. 1 (here) the net does depend on the loading, because the kinematic conditions concern only two points,

Table 1 Comparison of the normalized total volumes $V / V_{0}$, where $V_{0}=P L / \sigma_{p}$

\begin{tabular}{lllllr}
\hline & & \multicolumn{2}{c}{ LP-IPM $^{\mathrm{a}}$} & \multicolumn{2}{c}{ SA $^{\mathrm{b}}$} \\
\cline { 3 - 6 } Problem type & Exact vol. & Num. vol. & Rel. err. (\%) & Num. vol. \\
\hline Fig. 1a & 3.77092 & 3.77509 & 0.11 & 4.66312 & 23.66 \\
Fig. 2a & 4.63821 & 4.64084 & 0.06 & 4.66312 & 0.54 \\
Fig. 3a & 3.67773 & 3.68115 & 0.09 & 3.71506 & 1.02 \\
\hline
\end{tabular}

${ }^{\text {a }}$ Results by the program of Sokół (2011, cf. the Electronic Supplementary Material—ESM) based on the linear programming formulation and the interior point method

${ }^{\mathrm{b}}$ Results reported in Vázquez Espí and Cervera Bravo (2011), found by the simulated annealing method 
i.e. the fixed hinge supports. Therefore, the exact solution cannot be constructed in two subsequent steps, as in the case of the cantilevers: by starting from finding the Hencky net and then by solving the static problem. Here the kinematic and static problems are coupled, although no constitutive relations link the internal forces with virtual strains. They are replaced by Michell's optimality conditions. The current topic is also associated with the question of the division of Michell problems into those for which the superposition principle holds and the optimal layouts are immersed in a fixed Hencky net (the layout concerning a system of loads is a simple sum of layouts relevant to subsequent point loads); and other kind of Michell's problems for which such a superposition does not hold. This question has not been as yet resolved in a systematic way. We know, however, that the class of solutions considered in this paper belongs to the class not characterized by such a superposition.

Finally, it is worth reminding that Michell's problem can be formulated in two forms: in terms of internal forces (or averaged stresses or stress resultants-not in terms of stresses simply, since the stress level is fixed!) and in terms of virtual kinematic fields, and-that these two problems are equivalent as being mutually dual, see Rozvany (1976), and Strang and Kohn (1983). These two problems are named $(\mathrm{P})$, Section 2 and $\left(\mathrm{P}^{*}\right)$ in Section 3 of Strang and Kohn (1983). In the problem $\left(\mathrm{P}^{*}\right)$ the virtual displacements should be kinematically admissible and the relevant strains should belong to a certain locking locus, cf. Lewiński (2004). In the known Michell-like solutions the feasible domain is divided into two kinds of regions: in some region (can be multi-connected) the virtual strain field vanishesnote that zero strain belongs to the interior of the locking locus - and in the remaining region the virtual strain lies on the boundary of the locking locus. The minimizer of problem $(\mathrm{P})$, i.e. the internal force tensor field is linked with the maximizer of problem $\left(\mathrm{P}^{*}\right)$, i.e. the virtual displacement vector field, by the conditions (18) in Strang and Kohn (1983). For the truss-like problem they assume the form of the Karush-Kuhn-Tucker conditions and, consequently, they are both necessary and sufficient conditions of optimality. This contradicts the statement from the Abstract of the Discussion: "the Michell theorem is a sufficient test ... but maybe not necessary". Let us stress here: it is necessary for correctness of the optimum solution.

Acknowledgments The paper was prepared within the Research Grant no N506 071338, financed by the Polish Ministry of Science and Higher Education, entitled: Topology Optimization of
Engineering Structures. Simultaneous shaping and local material properties determination.

Open Access This article is distributed under the terms of the Creative Commons Attribution Noncommercial License which permits any noncommercial use, distribution, and reproduction in any medium, provided the original author(s) and source are credited.

\section{References}

Chan HSY (1975) Symmetric plane frameworks of least weight. In: Sawczuk A, Mróz Z (eds) Optimization in structural design. Springer-Verlag, Berlin, Heidelberg, New York, pp 313-326

Cox HL (1965) The design of structures of least weight. Pergamon, Oxford

Hemp WS (1973) Optimum structures. Clarendon, Oxford

Lewiński T (2004) Michell structures formed on surfaces of revolution. Struct Multidisc Optim 28:20-30

Maxwell C (1870) On reciprocal figures, frames and diagrams of forces. Trans Roy Soc of Edinburgh, Scientific Papers II 26:161207

McConnel RE (1974) Least-weight frameworks for loads across span. J Eng Mech Div 100:885-901

Michell AGM (1904) The limits of economy of material in frame structures. Phil Mag 8:589-597

Rozvany GIN (1976) Optimal design of flexural systems. Pergamon, London

Rozvany GIN (2011) On symmetry and non-uniqueness in exact topology optimization. Stuct Multidisc Optim 43:297-317

Sokół T (2011) A 99 line code for discretized Michell truss optimization written in Mathematica. Struct Multidisc Optim 43:181-190

Sokół T, Lewiński T (2010) On the solution of the three forces problem and its application in optimal designing of a class of symmetric plane frameworks of least weight. Struct Multidisc Optim 42:835-853

Sokół T, Lewiński T (2011) On the three forces problem in truss topology optimization. Analytical and numerical solutions. 9th World Congress on Structural and Multidisciplinary Optimization, WCSMO-9, 13-17 June, 2011, Shizuoka, Japan, Book of Abstract p 76, Full paper on CD

Sokół T, Rozvany GIN (2011) Important new results in exact topology optimization. In: Borkowski, Lewiński, Dzierżanowski (eds) Proc. of 19th int. conf. On "Computer Methods in Mechanics", CMM 2011, 09-12-May, Warsaw, pp 459-460. ISBN: 978-83-7207-943-5

Sokol T, Rozvany GIN (2012) New analytical benchmarks for topology optimization and their implications, Part I: bi-symmetric trusses with two point loads between supports. Struct Multidisc Optim (in press)

Strang G, Kohn RV (1983) Hencky-Prandtl nets and constrained Michell trusses. Comput Methods Appl Mech Eng 36:207222

Vázquez Espí M, Cervera Bravo J (2011) On the solution of the three forces problem and its application in optimal designing of a class of symmetric plane frameworks of least weight. Discussion on the Michell class concept. Struct Multidisc Optim doi:10.1007/s00158-011-0702-3 